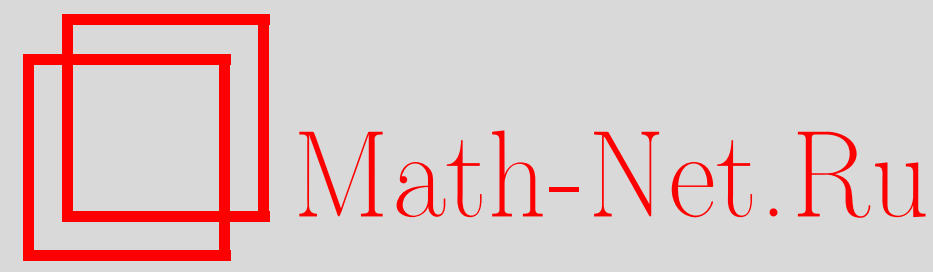

Ю. Г. Леонов, Об оценке снизу роста 3-порожденной 2-группы, Матем. сб., 2001, том 192, номер 11, 77-92

DOI: https://doi.org/10.4213/sm610

Использование Общероссийского математического портала Math-Net.Ru подразумевает, что вы прочитали и согласны с пользовательским соглашением

http: //www.mathnet.ru/rus/agreement

Параметры загрузки:

IP : 54.89 .56 .158

26 апреля 2023 г., 15:12:33 


\title{
Об оценке снизу роста 3-порожденной 2-группы
}

\begin{abstract}
В работе найдена нетривиальная оценка снизу роста одной периодической 3 -порожденной группы. Это позволяет отрицательно ответить на предположение Р.И. Григорчука, которое было сформулировано в 1990 году.

Библиография: 13 названий.
\end{abstract}

\section{§1. Введение}

Первые примеры периодических не локально конечных групп были построены Е. С. Голодом в 1964 году и С. В. Алешиным в 1972 году. Одной из самых простых таких групп является 2-группа, предложенная в 1980 году Р. И. Григорчуком [1]. Эта группа обладает многими интересными свойствами (см. [2], [3]), одним из которых является ее промежуточный рост.

Напомним, что функция роста конечно порожденной группы $H$ с системой порождаюших $S$ определяется соотношением

$$
\gamma(n)=\#\left\{g \in H ; l_{S}(g) \leqslant n\right\},
$$

где $l_{S}(g)$ - длина элемента $g$ относительно $S$.

Будем говорить, что функция $f_{1}(n)$ растет не быстрее, чем $f_{2}(n): f_{1}(n) \preceq f_{2}(n)$, если найдется $c>0$ такое, что $f_{1}(n) \leqslant f_{2}(c n)$ для любых $n \in \mathbb{N}$. Если $f_{1}(n) \preceq$ $f_{2}(n)$ и $f_{2}(n) \preceq f_{1}(n)$, то функции эквивалентны: $f_{1}(n) \sim f_{2}(n)$. Функции роста одной и той же конечно порожденной группы при различных конечных системах порождающих эквивалентны.

В 1968 году Дж. Милнор [4] поставил вопрос о сушествовании групп, у которых функция роста растет быстрее любой степенной и медленнее показательной. Такие группы называются группами промежуточного роста.

В работе [5] Р. И. Григорчук показал, что группа $G$ из [1] имеет промежуточньй рост, а именно справедливы неравенства:

$$
e^{\sqrt{n}} \preceq \gamma_{G}(n) \preceq e^{n^{\log _{32} 31}},
$$

тем самым ответив на вопрос Милнора.

Продолжая исследовать шкалу роста конечно порожденных групп, Р.И. Григорчук установил в работе [6], что если группа $H$ аппроксимируется конечными $p$-группами и допускает оценку роста

$$
\gamma_{H}(n) \prec e^{\sqrt{n}}
$$

(т.е. $\gamma_{H}(n) \preceq e^{\sqrt{n}}$ и не выполняется $\left.\gamma_{H}(n) \succeq e^{\sqrt{n}}\right)$, то $H$ почти нильпотентна.

Автор благодарит Swiss National Fund (Женева, Швейцария) за частичную поддержку в работе.

$$
\text { (C) Ю.Г. ЛЕОнов } 2001
$$


Таким образом, для конечно порожденных финитно аппроксимируемых групп мы имеем шкалу степеней роста групп с лакуной между степенными функциями и функцией $e^{\sqrt{n}}$; причем Р.И. Григорчуком высказана гипотеза о том, что эта лакуна имеет место даже для всего класса конечно порожденных групп.

В связи с этим возник вопрос о существовании группы с функцией роста, эквивалентной $e^{\sqrt{n}}$. В работе [6] установлено, что такие группы при условии аппроксимации их конечньпи $p$-группами имеют конечную ширину (т.е. ранги факторов нижнего центрального ряда равномерно ограничены). Групшы конечной ширины - это интересный и важный класс групा [7], и из известных конечно порожденных групп промежуточного роста группа $G$ является пока единственным примером группы конечной шпиины (другие примеры подобного рода из [8] родственны этой группе).

В работе [9] поставлен вопрос: действительно ли эти группы имеют рост $e^{\sqrt{n}}$ ? В настоящей работе (ранее результат был анонсирован автором в [10]) мы отвечаем на этот вопрос отрицательно. А именно:

ТЕОРемА. Для группьи $G$ из [1] верна оценка

$$
\gamma_{G}(n) \succeq e^{n^{\alpha}}
$$

əде $\alpha=\log _{3.9} 2=0.5093 \ldots>\frac{1}{2}$.

Прежде чем сформулировать вспомогательные результаты, используемые при доказательстве теоремы, определим группу $G$, а также отметим основные ее свойства. Первоначально эта группа была задана как группа преобразований отрезка $[0,1]$, сохраняющих лебегову меру. Позже выяснилось, что она имеет много других, эквивалентных заданий, например как группа, порожденная конечньми автоматами, или как группа, действующая автоморфизмами бинарного дерева. Здесь нам удобно интерпретировать ее элементы как преобразования в пространстве $\Omega=\{0,1\}^{\mathbb{N}}$ бесконечных (вправо) последовательностей алфавита $\{0,1\}$.

А именно для любой последовательности $\alpha=\alpha_{0} \alpha_{1} \ldots$ определим преобразование $a$ по правилу

$$
a(\alpha)=\tau\left(\alpha_{0}\right) \alpha_{1} \ldots, \quad \text { где } \tau(x)= \begin{cases}0, & x=1 \\ 1, & x=0 .\end{cases}
$$

Пусть $\sigma(\alpha)=\alpha_{1} \alpha_{2} \ldots-$ сдвиг влево последовательности $\alpha$. Определим также преобразования $b, c, d$ по правилу: для любых $\alpha \in \Omega$ полагаем

$$
\begin{aligned}
& b(\alpha)= \begin{cases}0 a(\sigma(\alpha)), & \alpha_{0}=0, \\
1 c(\sigma(\alpha)), & \alpha_{0}=1,\end{cases} \\
& c(\alpha)= \begin{cases}0 a(\sigma(\alpha)), & \alpha_{0}=0 \\
1 d(\sigma(\alpha)), & \alpha_{0}=1\end{cases} \\
& d(\alpha)= \begin{cases}\alpha, & \alpha_{0}=0 \\
1 b(\sigma(\alpha)), & \alpha_{0}=1\end{cases}
\end{aligned}
$$

Группа $G$ порождается элементами $a, b, c, d$ с операцией суперпозиции преобразований. Очевидно, что $a^{2}=b^{2}=c^{2}=d^{2}=b c d=1$.

Пусть $H \leqslant G$ - подгруппа группы $G$, состоящая из элементов, оставляющих на месте первую букву любой последовательности $\alpha \in \Omega$. Легко видеть, что в $H$ 
входят те и только те слова группы $G$, которые имеют четное число букв $a$ в своей записи, и что $H=\langle b, c, d, a b a, a c a, a d a\rangle$. Рассмотрим вложение (мономорфизм) $\psi: H \rightarrow G \times G$, заданное на порождающих следующим образом:

$$
\begin{aligned}
& \psi(b)=(a, c), \quad \psi(c)=(a, d), \quad \psi(d)=(1, b), \\
& \psi(a b a)=(c, a), \quad \psi(a c a)=(d, a), \quad \psi(a d a)=(b, 1)
\end{aligned}
$$

(это вложение определено в [1]). Обозначим через $l(g)$ длину элемента $g \in H$ относительно системы порождающих $S=\{b, c, d, a b a, a c a, a d a\}$.

Распространим $\psi$ на всю группу $G$, положив $\psi(g)=\psi(g a)$ при $g \notin H$. Можно считать $\psi^{n}(g)$ упорядоченной последовательностью из $2^{n}$ элементов, определенной индукцией по правилу $\psi^{n}(g)=\left(\psi^{n-1}\left(g_{1}\right), \psi^{n-1}\left(g_{2}\right)\right)$, если $\left(g_{1}, g_{2}\right)=\psi(g)$. Из (1) легко следует, что при $\psi(g)=\left(g_{1}, g_{2}\right)$ выполняется $l\left(g_{i}\right)<l(g), i=0,1$, кроме случая, ког да $g$ или $g a-$ порождающий или единичный элемент. Таким образом, для любых $g \in G$ найдется $m$ такое, что любое слово в последовательности $\psi^{m}(g)$ имеет длину $\leqslant 1$. Наименьшее $m$ с таким свойством назовем глубиной и обозначим через $f(g)$. Важную роль в оценке роста играет функция $r: \mathbb{N} \cup\{0\} \rightarrow \mathbb{N} \cup\{0\}$, определенная следующим образом:

$$
r(n)=\max _{g, f(g) \leqslant n} l(g)
$$

Основньм утверждением при доказательстве нашей теоремы является

Лемма 1. Существует $Q<4$ такое, что для некоторой постоянной $L>0$ выполняется $r(n) \leqslant Q \cdot r(n-1)+L$ для любих $n \in \mathbb{N}$.

Покажем, как, используя лемму 1, получить утверждение теоремы. Рассмотрим множество $F(n)=\{g \in G ; f(g) \leqslant n\}$ и его мошность $\bar{F}(n)=\#\{g \in G$; $f(g) \leqslant n\}$. Можно показать (см. [11] или [12]), что

$$
\bar{F}(n)=|F(n)| \geqslant 2^{2^{n} \cdot c_{1}}, \quad 0<c_{1} \leqslant 1
$$

Из леммы 1 следует, что $r(n) \leqslant Q^{n} \cdot L^{\prime}, L^{\prime}$ - постоянная, и так как количество элементов длины $\leqslant r(n)$ не меньше, чем количество элементов в множестве $F(n)$, TO

$$
\gamma_{G}(r(n)) \geqslant|F(n)| \geqslant 2^{2^{n} \cdot c_{1}}
$$

Отсюда $\gamma_{G}(n) \succeq e^{n^{\alpha}}$, где $\alpha=\log _{Q} 2$, и эта оценка доказывает теорему.

Прежде чем переходить к доказательству леммы 1, сформулируем родственный, но намного более простой результат, известный (в иной терминологии) еще с работы $[5]$.

ПРЕДЛОЖЕНИЕ 1. Для любъх $n \in \mathbb{N}$ верно неравенство

$$
r(n) \leqslant 4 \cdot r(n-1)+\text { const. }
$$


ДокАЗАТЕЛЬСТво. Пусть $g \in H, \psi(g)=\left(g_{1}, g_{2}\right)$. Покажем, что

$$
l(g) \leqslant 2\left(l\left(g_{1}\right)+l\left(g_{2}\right)\right)+\text { const. }
$$

Зафиксируем приведенные слова $g_{1}$ и $g_{2}$. При $x \in\{b, c, d\}$ обозначим

$$
\varphi(x)= \begin{cases}c, & x=d, \\ d, & x=b, \\ b, & x=c .\end{cases}
$$

Рассмотрим начало слова $g_{1}$.

1. Для $g_{1}=x g_{1}^{\prime}$ имеем

$$
\psi(g)=\psi(a \varphi(x) a) \cdot\left(g_{1}^{\prime}, u_{x} g_{2}\right), \quad l(g)=1+l\left(\left(g_{1}^{\prime}, u_{x} g_{2}\right)\right),
$$

где

$$
u_{x}= \begin{cases}a, & \text { если } x=c \text { или } d, \\ 1, & x=b .\end{cases}
$$

2. Пусть $g_{1}=x^{a} g_{1}^{\prime}$. Тогда $\psi(g)=\left(x^{a} g_{1}^{\prime},\left(u_{x}^{d}\right)^{2} g_{2}\right)=\left(x^{a}, u_{x}^{d}\right) \cdot\left(g_{1}^{\prime}, u_{x}^{d} g_{2}\right)=$ $\psi\left(c \varphi(x)^{a} c\right) \cdot\left(g_{1}^{\prime}, u_{x}^{d} g_{2}\right)$ и $l(g) \leqslant 3+l\left(\left(g_{1}^{\prime}, u_{x}^{d} g_{2}\right)\right)$.

Так как варианты 1 и 2 чередуются, то после $n$-кратного их применения для слова $g_{1}$ имеем

$$
l(g) \leqslant n+3 n+l\left(\left(g_{1}^{\prime \prime}, h g_{2}\right)\right), \quad \text { где } h \in\left\langle a^{d}, a\right\rangle,
$$

при этом $l\left(g_{1}^{\prime \prime}\right)=l\left(g_{1}\right)-2 n, l(h) \leqslant$ const. Отсюда, достигнув достаточно больших $n$ (а именно $n>|a d|=4$ ) с условием $0 \leqslant l\left(g_{1}\right)-2 n \leqslant 1$, имеем $l(g) \leqslant 2 \cdot 2 n+$ $l\left(\left(g_{1}^{\prime \prime}, h g_{2}\right)\right) \leqslant 2 \cdot 2 n+2\left(l\left(g_{1}\right)-2 n\right)+2 \cdot l\left(\left(\widetilde{g}_{1}, h g_{2}\right)\right)=2 \cdot l\left(g_{1}\right)+2 \cdot l\left(\left(\widetilde{g}_{1}, h g_{2}\right)\right)$, $l\left(\widetilde{g}_{1}\right), l(h) \leqslant$ const.

Далее мы можем по аналогии повторить наши рассуждения для слова $h g_{2}$ и получить неравенство $l(g) \leqslant 2 \cdot l\left(g_{1}\right)+2 \cdot l\left(g_{2}\right)+2 \cdot l\left(\left(\widetilde{g}_{1}, \widetilde{g}_{2}\right)\right)$. Последнее слагаемое заведомо ограничено, и предложение доказано.

Для $\psi(g)=\left(g_{1}, g_{2}\right)$ обозначим $\psi\left(g_{1}\right)=\left(g_{1,1}, g_{1,2}\right), \psi\left(g_{2}\right)=\left(g_{1,3}, g_{1,4}\right)$. Далее по индукции рассматриваем $\psi\left(g_{i, 1}\right)=\left(g_{i+1,1}, g_{i+1,2}\right), \ldots, \psi\left(g_{i, k}\right)=\left(g_{i+1,2 k-1}\right.$, $\left.g_{i+1,2 k}\right)$. Тогда имеем

СлЕДСТВИЕ. Для $g \in G$ и для любых натуральных $m$ выполняется

$$
l(g) \leqslant 2^{m} \sum_{i=1}^{2^{m}} l\left(g_{m, i}\right)+\operatorname{const}(m) .
$$

\section{§2. Доказательство леммы 1}

Для некоторых $\varepsilon, \mu \in(0,1)$ рассмотрим множества элементов групшы $G$ глубины $\leqslant n:$

$$
\begin{aligned}
F_{\varepsilon}^{*}(n) & =\left\{g \in F(n) ; \psi(g)=\left(g_{1}, g_{2}\right), l\left(g_{i}\right) \leqslant \varepsilon \cdot l\left(g_{j}\right), i \neq j\right\}, \\
F_{\mu}^{* *}(n) & =\left\{g \in F(n) ; \psi(g)=\left(g_{1}, g_{2}\right), g_{i} \in F_{\mu}^{*}(n-1) \text { для некоторого } i \in\{1,2\}\right\} .
\end{aligned}
$$

Обозначим

$$
R(n)=\{g \in F(n) ; l(g)=r(n)\}
$$


Доказательство леммы разобьем на 3 части и выберем наихудшую оценку для коэффициента $Q$.

I. Допустим, $g \in F_{1 / 2}^{*}(n) \cap R(n), \psi(g)=\left(g_{1}, g_{2}\right)$. Тогда по предложению 1

$$
r(n)=l(g) \leqslant 2 \cdot\left(l\left(g_{1}\right)+l\left(g_{2}\right)\right) \leqslant 2 \cdot\left(r(n-1)+\frac{1}{2} \cdot r(n-1)\right)=3 \cdot r(n-1) .
$$

II. Пусть $g \in F_{1 / 6}^{* *}(n) \cap R(n), \psi(g)=\left(g_{1}, g_{2}\right)$. Снова используя предложение 1 , имеем

$$
r(n)=l(g) \leqslant 4 \cdot\left(3 \cdot r(n-2)+\frac{1}{6} \cdot r(n-2)\right)=\frac{38}{3} \cdot r(n-2) .
$$

Если это неравенство окажется наихудшим среди наших трех случаев, то можно утверждать, что

$$
r(n) \leqslant\left(\sqrt{\frac{38}{3}}\right)^{n}
$$

т.е. $Q$ можно взять равным $\sqrt{38 / 3}<4$.

III. Оставшийся случай рассмотрим в виде леммы.

Лемма 2. Пусть $g \in F(n) \backslash\left(F_{1 / 2}^{*}(n) \cup F_{1 / 6}^{* *}(n)\right), \psi(g)=\left(g_{1}, g_{2}\right) . \quad$ Тогда найдутся $\lambda<2$ и $L_{1} \in \mathbb{R}$ такие, что справедливо неравенство

$$
l(g) \leqslant \lambda\left(l\left(g_{1}\right)+l\left(g_{2}\right)\right)+L_{1} .
$$

Заметим, что из (2) следует утверждение леммы 1. Действительно, если $g \in$ $R(n)$, то имеем $r(n) \leqslant \lambda(r(n-1)+r(n-1))+L_{1}$ и в лемме можно выбрать $Q=\max \{2 \lambda, \sqrt{38 / 3}\}$.

ДокАЗАТЕЛЬСТво ЛЕммЫ 2. Наша основная цель - одновременно используя слова $g_{1}$ и $g_{2}$, конструировать некоторый графический вид слова $g$, показывая, что его длина "не слишком большая". А именно в зависимости от нескольких первых букв в словах $g_{1}$ и $g_{2}$ мы будем выбирать слово $v_{1} \in G$ с условием

$$
\left(g_{1}, g_{2}\right)=\psi\left(v_{1}\right) \cdot\left(g_{1}^{(1)}, g_{2}^{(1)}\right), \quad l\left(g_{1}^{(1)}\right)+l\left(g_{2}^{(1)}\right)<l\left(g_{1}\right)+l\left(g_{2}\right) .
$$

Продолжив наш процесс уже для слова $\left(g_{1}^{(1)}, g_{2}^{(1)}\right)$ и т. д., мы получим:

$$
\left(g_{1}, g_{2}\right)=\psi\left(v_{1} v_{2} \cdots v_{k}\right) \cdot\left(g_{1}^{(k)}, g_{2}^{(k)}\right) .
$$

Если при росте $k$ мы покажем, что

$$
l\left(v_{1} v_{2} \cdots v_{k}\right) \leqslant \lambda\left(l\left(g_{1}\right)-l\left(g_{1}^{(k)}\right)+l\left(g_{2}\right)-l\left(g_{2}^{(k)}\right)\right)+\mathrm{const}
$$

для некоторого $\lambda<2$, то требование (2) будет выполняться.

Слова $v_{1}, v_{2}, \ldots$, которые будут нами однозначно определяться по словам $g_{1}$ и $g_{2}$, мы будем называть факторами.

Положим $x_{1}, x_{2}, \ldots$ и $y_{1}, y_{2}, \ldots \in\{b, c, d\}$. Рассмотрим в зависимости от графического вида приведенных слов $g_{1}$ и $g_{2}$ следуюшие случаи.

$c 1 . g_{1}=a x_{1} \ldots, g_{2}=y_{1} a \ldots$ Этот случай разобьем на два:

c1.1. $y_{1} \neq b$,

c1.2. $y_{1}=b$. 
Аналогично разбиваем случай

$c 2 . g_{1}=x_{1} a \ldots, g_{2}=a y_{1} \ldots$ на

c2.1. $x_{1} \neq b$,

c2.2. $x_{1}=b$.

Наконец, рассмотрим самњй интересный вариант

$c 3 . g_{1}=a x_{1} a x_{2} \ldots, g_{2}=a y_{1} a y_{2} \ldots$ и следуюшие его разбиения:

c3.1.1. $x_{1}=b$,

c3.1.2. $y_{1}=b$ (при $x_{1}=y_{1}=b$ есть возможность выбирать один из случаев $c 3.1 .1$ или $c 3.1 .2)$;

c3.2.1. $y_{1} \neq b, x_{1}=d$,

$c 3.2 .2 . y_{1}=d, x_{1}=c$;

c3.3. $x_{1}=c, y_{1}=c$.

Последний вариант является, как выяснится ниже, самым содержательным, и его требуется разбить на ряд подвариантов:

c3.3.1. $y_{2}=b$. Этот вариант разобьем еше на три:

c3.3.1.1. $x_{2}=b$,

c3.3.1.2. $x_{2}=d$,

c3.3.1.3. $x_{2}=c$;

c3.3.2. $y_{2}=d$,

c3.3.3. $y_{2}=c$.

Сказанное для наглядности представим в таблице 1.

ТАБЛИЦА 1

\begin{tabular}{|l|l|l|}
\hline Начало слова $g_{1}$ & Начало слова $g_{2}$ & Вариант \\
\hline$a \ldots$ & $\{c, d\} a \ldots$ & $c 1.1$ \\
\hline$a \ldots$ & $b a \ldots$ & $c 1.2$ \\
\hline$\{c, d\} a \ldots$ & $a \ldots$ & $c 2.1$ \\
\hline$b a \ldots$ & $a \ldots$ & $c 2.2$ \\
\hline$a b \ldots$ & $a \ldots$ & $c 3.1 .1$ \\
\hline$a \ldots$ & $a b \ldots$ & $c 3.1 .2$ \\
\hline$a d \ldots$ & $a\{c, d\} \ldots$ & $c 3.2 .1$ \\
\hline$a c \ldots$ & $a d \ldots$ & $c 3.2 .2$ \\
\hline$a c a b \ldots$ & $a c a b \ldots$ & $c 3.3 .1 .1$ \\
\hline$a c a d \ldots$ & $a c a b \ldots$ & $c 3.3 .1 .2$ \\
\hline$a c a c \ldots$ & $a c a b \ldots$ & $c 3.3 .1 .3$ \\
\hline$a c \ldots$ & $a c a d \ldots$ & $c 3.3 .2$ \\
\hline$a c \ldots$ & $a c a c \ldots$ & $c 3.3 .3$ \\
\hline
\end{tabular}

Заметим, что еще возможен случай

$c 4 . g_{1}=x_{1} a \ldots, g_{2}=y_{1} a \ldots$.

Однако мы проведем рассуждения таким образом, чтобы не учитывать этот вариант.

Множество наших случаев обозначим через $C$. Будем говорить, что слово $g$ относится к случаю $\bar{c}$ и писать $g \in \bar{c}$, если для преобразования слова $g$ необходимо использовать вариант $\bar{c} \in C$.

Пусть $g_{1}=(a) x_{1} a x_{2} \ldots, g_{2}=(a) y_{1} a y_{2} \ldots$ (элемент в круглых скобках может отсутствовать). 
ТАБЛИЦА 2

\begin{tabular}{|l|l|}
\hline Случай & Фактор \\
\hline$c 1.1, c 1.2$ & $y_{1}$ \\
\hline$c 2.1, c 2.2$ & $x_{1}^{a}$ \\
\hline$c 3.1 .1, c 3.2 .2$ & $c x_{1}^{a} c$ \\
\hline$c 3.1 .2, c 3.2 .1$ & $c^{a} y_{1} c^{a}$ \\
\hline$c 3.3 .2$ & $c^{a} b c^{a} c$ \\
\hline$c 3.3 .3$ & $c^{a} b c^{a} b c^{a}$ \\
\hline$c 3.3 .1 .1$ & $c b^{a} c d^{a}$ \\
\hline$c 3.3 .1 .2$ & $c b^{a} c c^{a}$ \\
\hline$c 3.3 .1 .3$ & $c b^{a} c b^{a} c$ \\
\hline
\end{tabular}

Предлагаемые нами факторы для каждого случая представлены в таблице 2.

Допустим, $g=v \bar{g}$, где $v$ - фактор, соответствующий элементу $\psi(g)=\left(g_{1}, g_{2}\right)$. Обозначим

$$
\operatorname{lose}_{1}(g)=l(g)-l(\bar{g}), \quad \operatorname{lose}_{2}(g)=l_{1}(g)-l_{1}(\bar{g}) .
$$

Пару $\left[\operatorname{lose}_{1}(g), \operatorname{lose}_{2}(g)\right]$ назовем параметром элемента $g$ и обозначим через $\operatorname{par}(\bar{c})$, где $\bar{c}-$ это случай, к которому относится элемент $g$. Так как фактор был взят с учетом случая $\bar{c}$ и параметры $\operatorname{lose}_{1}(g)$ и $\operatorname{lose}_{2}(g)$ (как будет видно ниже) определяются благодаря $v$ однозначно, то обозначение корректно.

Для ситуаций $c 1.1, c 1.2, \ldots$ будем обозначать относящиеся к ним факторы через $v 1.1, v 1.2, \ldots$ Рассмотрим элемент $\bar{g}$, когда $\psi(g)=\left(g_{1}, g_{2}\right)$ своим видом удовлетворяет одному из вышеперечисленных случаев. Ниже для наглядности будем иногда отождествлять элемент $g$ группы $G$ и его отображение $\psi(g)$.

$c 1.1$. Нетрудно видеть, что

$$
g=v 1.1 \cdot \bar{g}=y_{1} \bar{g}=y_{1}\left(x_{1} a \ldots, a y_{2} \ldots\right),
$$

$\bar{g} \in c 2$ и $\operatorname{par}(c 1.1)=[1,1]$.

$c 1.2 . g=v 1.2 \cdot \bar{g}=y_{1} \bar{g}=y_{1}\left(a x_{1} \ldots, a y_{2} \ldots\right), \bar{g} \in c 3, \operatorname{par}(c 1.2)=[1,1]$.

Далее имеем

$c 2.1 . g=v 2.1 \cdot \bar{g}=x_{1}^{a} \bar{g}=x_{1}^{a}\left(a x_{2} \ldots, y_{1} a \ldots\right), \bar{g} \in c 1, \operatorname{par}(c 2.1)=[1,1]$.

$c 2.2 . g=v 2.2 \cdot \bar{g}=x_{1}^{a} \bar{g}=x_{1}^{a}\left(a x_{2} \ldots, a y_{1} \ldots\right), \bar{g} \in c 3, \operatorname{par}(c 2.2)=[1,1]$.

Наконец, разберем подслучаи случая $c 3$.

c3.1.1. $g=\left(a_{b a x} \ldots, a y_{1} \ldots\right)=v 3.1 .1 \cdot \bar{g}=c d^{a} c \cdot \bar{g}=(a b a, 1) \cdot \bar{g}, \bar{g}=$ $\left(x_{2} a \ldots, a y_{1} \ldots\right) \in c 2, \operatorname{par}(c 3.1 .1)=[3,1]$.

c3.1.2. $g=\left(a x_{1} \ldots, a b a y_{2} \ldots\right)=v 3.1 .2 \cdot \bar{g}=c^{a} d c^{a} \cdot \bar{g}=(1, a b a) \cdot \bar{g}, \bar{g}=$ $\left(a x_{1} \ldots, y_{2} a \ldots\right) \in c 1, \operatorname{par}(c 3.1 .2)=[3,1]$.

$c 3.2 .1 . g=\left(\operatorname{adax}_{2} \ldots, a y_{1} \ldots\right)=v 3.2 .1 \cdot \bar{g}=c^{a} y_{1} c^{a} \cdot \bar{g}=\left(d a d, a y_{1} a\right) \cdot \bar{g}$.

Так как $|d a|=4$, то $\bar{g}=\left(\operatorname{adax}_{2} a \ldots, y_{2} a \ldots\right)$. Сокращения продолжаются, если $y_{2}=d$, но этот случай невозможен, иначе слово $g_{1}$ имеет начало adad $=d a d a$ и вопреки предположению не является приведенньм. Итак, $\bar{g} \in c 1, \operatorname{par}(c 3.2 .1)=$ $[3,2]$.

c3.2.2. $g=\left(\right.$ acax $\left._{2} \ldots, a_{a d a y_{2}} \ldots\right)=v 3.2 .2 \cdot \bar{g}=c b^{a} \mathrm{c} \cdot \bar{g}=($ aca,$d a d) \cdot \bar{g}$.

Аналогично предыдущему случаю $\bar{g}=\left(x_{2} a \ldots, a d y_{2} a y_{3} \ldots\right) \in c 2, \operatorname{par}(c 3.2 .2)=$ $[3,2]$.

Осталось рассмотреть преобразования вариантов случая $c 3.3$. 
c3.3.2. $g=\left(\right.$ acax $_{2} \ldots$, axaday $\left._{3} \ldots\right)=v 3.3 .2 \cdot \bar{g}=c^{a} b c^{a} c \cdot \bar{g}=($ dada,$a c a d) \cdot \bar{g}$, $\bar{g}=\left(\right.$ dabax $\left._{2} \ldots, a_{3} \ldots\right) \in c 2.1$. Имеем $\operatorname{par}(c 3.3 .2)=[4,1]$.

c3.3.3. $g=\left(\right.$ acax $_{2} \ldots$, acacay $\left._{3} \ldots\right)=v 3.3 .3 \cdot \bar{g}=c^{a} b c^{a} b c^{a} \cdot \bar{g}=($ dadad, acaca $) \cdot \bar{g}$, $\bar{g}=\left(a b a x_{2} \ldots, y_{3} a \ldots\right) \in c 1$. При этом $\operatorname{par}(c 3.3 .3)=[5,2]$.

c3.3.1.1. $g=\left(\operatorname{acabax}_{3} \ldots, \operatorname{acabay}_{3} \ldots\right)=v 3.3 .1 .1 \cdot \bar{g}=c b^{a} c d^{a} \cdot \bar{g}=($ baca, dad $) \cdot \bar{g}$,

$$
\bar{g}=\left(a_{3} \ldots, \text { dadacay }_{2} \ldots\right)=\left(\text { ax }_{3} \ldots, \text { adabay }_{2} \ldots\right) \in\left\{\begin{array}{l}
c 3.1 .1, x_{3}=b \\
c 3.2 .1, x_{3}=d \\
c 3.2 .2, x_{3}=c
\end{array}\right.
$$

$\operatorname{par}(c 3.3 .1 .1)=[4,1]$.

c3.3.1.2. $g=\left(\right.$ acadax $_{3} \ldots$, acabay $\left._{3} \ldots\right)=v 3.3 .1 .2 \cdot \bar{g}=c b^{a} c c^{a} \cdot \bar{g}=($ daca,$a d a d) \cdot \bar{g}$, $\bar{g}=\left(a_{3} \ldots\right.$, dababay $\left._{3} \ldots\right) \in c 1.1, \operatorname{par}(c 3.3 .1 .2)=[4,1]$.

c3.3.1.3. $g=\left(\right.$ acacax $_{3} \ldots$, acabay $\left._{3} \ldots\right)=v 3.3 .1 .3 \cdot \bar{g}=c b^{a} c b^{a} c \cdot \bar{g}=($ acaca, dadad $)$. $\bar{g}, \bar{g}=\left(x_{3} a \ldots\right.$, ababay $\left._{3} \ldots\right) \in c 2, \operatorname{par}(c 3.3 .1 .3)=[5,2]$.

Подведем итог сказанному вьше, собрав наши рассуждения в таблицу 3.

ТАБЛИЦА 3

\begin{tabular}{|l|l|c|}
\hline Вариант & Переход к варианту & Параметр перехода \\
\hline$c 1.1$ & $c 2$ & {$[1,1]$} \\
\hline$c 1.2$ & $c 3$ & {$[1,1]$} \\
\hline$c 2.1$ & $c 1$ & {$[1,1]$} \\
\hline$c 2.2$ & $c 3$ & {$[1,1]$} \\
\hline$c 3.1 .1$ & $c 2$ & {$[3,1]$} \\
\hline$c 3.1 .2$ & $c 1$ & {$[3,1]$} \\
\hline$c 3.2 .1$ & $c 1$ & {$[3,2]$} \\
\hline$c 3.2 .2$ & $c 2$ & {$[3,2]$} \\
\hline$c 3.3 .1 .1$ & $c 3.1 .1, c 3.2 .1, c 3.2 .2$ & {$[4,1]$} \\
\hline$c 3.3 .1 .2$ & $c 1.1$ & {$[4,1]$} \\
\hline$c 3.3 .1 .3$ & $c 2$ & {$[5,2]$} \\
\hline$c 3.3 .2$ & $c 2.1$ & {$[4,1]$} \\
\hline$c 3.3 .3$ & $c 1$ & {$[5,2]$} \\
\hline
\end{tabular}

Еще раз отметим, что слово $g=\left(g_{1}, g_{2}\right)$ при фиксированных (приведенных) словах $g_{1}$ и $g_{2}$ однозначно определяет случай $c$, который, в свою очередь, определяет фактор $v$. Далее мы однозначно определяем, к какому случаю относится слово $\bar{g}$, и можем применить текущие рассуждения для него. Таким образом, левая часть слова $g$ от его начала и далее однозначно определяет последовательность случаев и последовательность факторов, которые мы применяем согласно этим случаям.

Пусть дано слово $g=\left(g_{1}, g_{2}\right) \in H$ и $\bar{c}=c_{1}, \ldots, c_{r}$ - последовательность случаев. Обозначим $\operatorname{par}\left(c_{i}\right)=\left[l_{i, 1}, l_{i, 2}\right]$. Тогда пару $\left[l_{1,1}+\cdots+l_{r, 1}, l_{1,2}+\cdots+l_{r, 2}\right]$ назовем параметром последовательности случаев $\bar{c}$ и обозначим через $\operatorname{par}(\bar{c})$. Если $\operatorname{par}(\bar{c})=\left[u_{1}, u_{2}\right]$, то отношение $u_{1} / u_{2}$ обозначим через $\lambda(\bar{c})$. Для облегчения понимания процесса преобразований элемент $\bar{g}$, полученный из элемента $g$ в случае (в последовательности случаев) $\bar{c}$, обозначим через $g(\bar{c})$.

Прежде чем рассматривать возможные последовательности факторов и их параметры, сделаем несколько уточнений, касающихся сокращений, возникающих при переходе от одного варианта к другому. 
Например, при переходе от варианта $c 1.2$ к варианту $c 3.1 .1$ мы пользуемся фактором $d$, а от варианта $c 3.1 .1$ далее - фактором $c d^{a} c$. Таким образом, истинная длина фактора $v 1.2 \cdot v 3.1 .1$ меньше чем сумма длин наших факторов. Это замечание позволяет нам заменить параметр $\operatorname{par}(c 1.2)=[1,1]$ при условии, что мы переходим к случаю $c 3.1 .1$, на параметр $\operatorname{par}_{c 3.1 .1}(c 1.2)=[0,1]$. Искусственное уменьшение параметра длины слова $v 1.2$ при условии перехода к данному варианту $c 3.1 .1$ производится нами для удобства и является корректным.

По той же причине можно уменьшить и некоторые другие параметры, считая

$$
\operatorname{par}_{c 3.2 .2}(c 1.2)=\operatorname{par}_{c 3.3 .1 .1}(c 1.2)=\operatorname{par}_{c 3.3 .1 .2}(c 1.2)=\operatorname{par}_{c 3.3 .1 .3}(c 1.2)=[0,1] \text {. }
$$

Аналогично

$$
\operatorname{par}_{c 3.1 .2}(c 2.2)=\operatorname{par}_{c 3.2 .1}(c 2.2)=\operatorname{par}_{3.3 .2}(c 2.2)=\operatorname{par}_{3.3 .3}(c 2.2)=[0,1] .
$$

Наконец, можно считать $\operatorname{par}_{c 3.2 .1}(c 3.3 .1 .1)=[3,1]$.

Рассматривая последовательности вариантов, удобно использовать диаграмму переходов от варианта к варианту, которая основана на данных рассуждениях и таблице 3 (см. рис. 1).

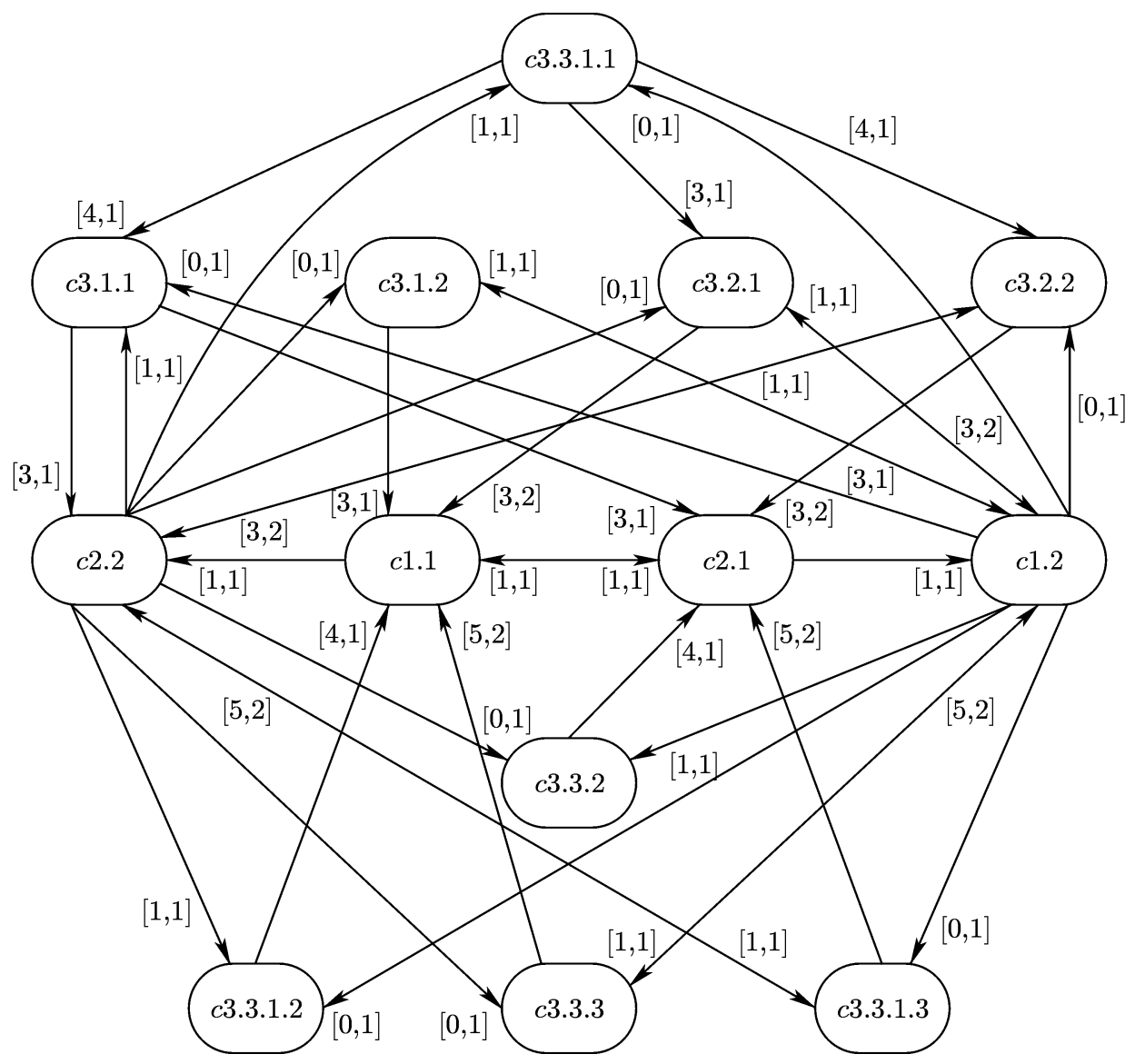

Рис. 1 
Будем говорить, что параметр $\left[u_{1}, u_{2}\right]$ больше параметра $\left[w_{1}, w_{2}\right]$, если $u_{1} / u_{2}>$ $w_{1} / w_{2}$. Кроме того, можно определить сумму параметров двух слов $g$ и $h$ как параметр слова $g h:\left[u_{1}, u_{2}\right]+\left[w_{1}, w_{2}\right]=\left[u_{1}+w_{1}, u_{2}+w_{2}\right]$.

Нашей основной задачей является доказательство оценки (2) с некоторым коэффициентом $\lambda<2$. Для этого, напротив, будем пытаться строить последовательности вариантов $c$ с условием $\operatorname{par}(c)=\left[u_{1}, u_{2}\right]$ и $u_{1} / u_{2} \geqslant 2$.

Начальньм вариантом каждой такой последовательности будет один случай из множества

$\{c 3.1 .1, c 3.1 .2, c 3.3 .2, c 3.3 .3, c 3.3 .1 .1, c 3.3 .1 .2, c 3.3 .1 .3\}$

что следует из их параметров.

1. Допустим, начальные фрагменты слов $g_{1}$ и $g_{2}$ удовлетворяют случаю $c 3.3 .2$. Продолжая рассматривать далее следующие случаи, мы неизменно получаем последовательность $\bar{c}=\{c 3.3 .2, c 2.1\}$ с параметром $\operatorname{par}(\bar{c})=[4,1]+[1,1]=[5,2]$. При этом параметр последовательности $\{c 3.3 .2, c 2.1, c 1.1\}$ равен $[6,3]$ и следующий переход (в зависимости от вида слов $g_{1}$ и $g_{2}$ ) произойдет или к $c 2.2$, или к $c 2.1$. В любом случае мы получим последовательность $c^{\prime}$ с параметром $\operatorname{par}\left(c^{\prime}\right)=[7,4]$ и $\lambda\left(c^{\prime}\right)=7 / 4<2$.

С другой стороны, для $c^{\prime}=\{c 3.3 .2, c 2.1, c 1.2\}$ имеем элемент $\bar{g}\left(c^{\prime}\right)=(a b \ldots$, $\left.y_{4} \ldots\right)$ и в дальнейшем переходим к варианту $c 3.1 .1$ с параметром $\operatorname{par}\left(c^{\prime}\right)=[4,1]+$ $[1,1]+\operatorname{par}_{c 3.1 .1}(c 1.2)=[5,3]$. Отсюда $\operatorname{par}\left(\left\{c^{\prime}, c 3.1 .1\right\}\right)=[5,3]+[3,1]=[8,4]$. Любой следующий после $c 3.1 .1$ вариант - $c 2.2$ или $c 2.1$ - имеет параметр $[1,1]$, и поэтому общий параметр получающейся последовательности будет равен $[9,5]$.

2. Допустим, наша последовательность начинается с варианта $c 3.3 .3$. При $\bar{c}=$ $\{c 3.3 .3, c 1.1\}$ параметр $\operatorname{par}(\bar{c})=[6,3]$, и далее после $c 1.1$ выполняется или $c 2.2$, или $c 2.1$, что дает параметр последовательности $[7,4]$.

Пусть $\bar{c}=\{c 3.3 .3, c 1.2\}$. После выполнения преобразований для данной последовательности случаев получаем $\bar{g}=\left(a b a x_{2} \ldots, y_{4} \ldots\right)$ и переходим к варианту $c 3.1 .1$. Отсюда параметр последовательности $\bar{c}$ равен $\operatorname{par}(\bar{c})=[5,2]+[0,1]=$ $[5,3]$ и любая последовательность вариантов с началом $c 3.3 .3$ удовлетворяет услоВИю (2).

3. Началом является случай $c 3.1 .2$. Для последовательности случаев $\bar{c}=$ $\{c 3.1 .2, c 1.1\}$ параметром является пара $[3,1]+[1,1]=[4,2]$, и далее рассуждения аналогичны первому абзацу предыдущего пункта. Пусть теперь $\bar{c}=\{c 3.1 .2, c 1.2\}$. После $c 1.2$ возможны подслучаи случая $c 3$. Учитывая сокрашения после $c 1.2$ при переходе к вариантам $c 3.1 .1, c 3.2 .2, c 3.3 .1 . x$ (о чем было сказано в начале этого параграфа), имеет смысл рассматривать продолжения для вариантов $c 3.1 .2, c 3.2 .1$, c3.3.2 и с 3.3 .3 .

Пусть $z$ - некоторая последовательность наших случаев. Последовательность вида $z, z, \ldots$ назовем ииклом. Последовательность случаев $c$ назовем особой, если в наших рассуждениях $\lambda(c)=2$ и на основе данной последовательности возможен цикл.

При переходе к варианту 33.1 .2 мы фактически возвращаемся к началу п. 3 и можем иметь в дальнейших рассуждениях цикл. Таким образом, имеем особую последовательность $z_{1}=\{c 3.1 .2, c 1.2\}$.

Другие варианты дают впоследствии $\lambda<2$. Действительно, $\operatorname{par}(\{\bar{c}, c 3.2 .1\})=$ $[4,2]+[3,2]=[7,4]$, а переходы к $c 3.3 .2$ и c3.3.3 равносильны (равносильность следует из того, что $\lambda(\bar{c})=2$ ) обозрению вариантов с соответствующими началами, которые полностью рассмотрены в пш. 1 и 2. 
4. Допуская, что вариантом начала слова $g=\left(g_{1}, g_{2}\right)$ является случай $c 3.3 .1 .2$, мы приходим к рассмотрению последовательности случаев $\bar{c}=\{c 3.3 .1 .2, c 1.1\}$.

4.1. Параметр последовательности $\{\bar{c}, c 2.1\}$ равен $[4,1]+[1,1]+[1,1]=[6,3]$. После случая $c 2.1$ возможно или $c 1.1$, или $c 1.2$. При любом продолжении параметр последовательности станет равным $[7,4]$.

4.2. $c^{\prime}=\{c 3.3 .1 .2, c 1.1, c 2.2\}$. Рассмотрим вид слов $g_{1}$ и $g_{2}$, необходимых для последовательности $c^{\prime}: g(c 3.3 .1 .2)=\left(a x_{3} \ldots, d a b y_{2} \ldots\right), g(\{c 3.3 .1 .2, c 1.1\})=$ $\left(x_{3} a \ldots, a b a y_{2} \ldots\right), g\left(c^{\prime}\right)=\left(a x_{4} \ldots, a b a y_{2} \ldots\right)$. После $c 2.2$ мы переходим к случаю $c 3$ и, как видно, далее переходим к $c 3.1 .2$. Отсюда $\operatorname{par}\left(c^{\prime}\right)=[4,1]+[1,1]+$ $[0,1]=[5,3]$.

5. Началом является случай $c 3.3 .1 .3$. Для последовательности $\bar{c}=\{c 3.3 .1 .3$, $c 2.1\}$ параметр $\operatorname{par}(\bar{c})=[5,2]+[1,1]=[6,3]$, следующим вариантом является $c 1.1$ или $c 1.2$, и мы получаем последовательность с параметром $[7,4]$.

Рассмотрим $\bar{c}=\{c 3.3 .1 .3, c 2.2\}$. Имеем $g(\bar{c})=\left(a x_{4} \ldots, a b y_{2} \ldots\right)$, следующий случай $-c 3.1 .2$ и, таким образом, параметр $\operatorname{par}(\bar{c})=[5,3]$ по аналогии с п. 4.2.

6. Допустим, начальным вариантом является $c 3.1 .1$. Для последовательности $\bar{c}=\{c 3.1 .1, c 2.1\}$ параметр $\operatorname{par}(\bar{c})$ равен $[4,2]$. Продолжая рассуждения аналогично первому абзацу предыдущего пункта, получаем параметр [5, 3].

Пусть $\bar{c}=\{c 3.1 .1, c 2.2\}$. Для следующих возможных вариантов $c 3.1 .2, c 3.2 .1$, $c 3.3 .2, c 3.3 .3$ коэффициент последовательности $\lambda<2$ согласно (3). Рассмотрим оставшиеся продолжения последовательности $\bar{c}$.

6.1. $c^{\prime}=\{\bar{c}, c 3.2 .2\}$. Здесь $\operatorname{par}\left(c^{\prime}\right)=[3,1]+[1,1]+[3,2]=[7,4]$.

6.2. Для $c^{\prime}=\{\bar{c}, c 3.3 .1 .2\}$ или $c^{\prime}=\{\bar{c}, c 3.3 .1 .3\}$ следует воспользоваться рассуждениями для последовательностей с началом $c 3.3 .1 .2$ и c3.3.1.3 соответственно, имея в виду, что $\operatorname{par}(\bar{c})=[4,2]$.

6.3. При переходе к варианту $c 3.1 .1$ возможна особая последовательность $z_{2}=$ $\{c 3.1 .1, c 2.2\}$.

Повторение последовательности $z n$ раз подряд обозначим через $[z]_{n}$. Отметим, что после $\left[z_{2}\right]_{n}\left(n\right.$-кратного $(n \in \mathbb{N})$ повторения последовательности $\left.z_{2}\right)$ возможен переход к варианту $c 3.3 .1 .1$, о котором идет речь ниже.

7. Рассмотрим оставшийся, самьй интересньй случай c3.3.1.1. Разобьем этот вариант на подварианты.

7.1. Пусть $\bar{c}=\{c 3.3 .1 .1, c 3.2 .2\}$. Если далее в последовательности идет случай $c 2.1$, то, учитьвая параметр $\operatorname{par}(\{\bar{c}, c 2.1\})=[4,1]+[3,2]+[1,1]=[8,4]$, можно применить рассуждения из первого абзаца п. 5 с последующим параметром $[9,5]$.

Для последовательности случаев $c^{\prime}=\{\bar{c}, c 2.2\}$ с параметром $\operatorname{par}\left(c^{\prime}\right)=[8,4]$ рассмотрим

$$
g\left(c^{\prime}\right)=\left(a x_{5} \ldots, \operatorname{acay}_{2} \ldots\right)
$$

Отсюда видно, что следующими вариантами могут быть $c 3.3, c 3.1 .1$ или $c 3.2 .1$.

Учитывая (3), продолжения $c 3.2 .1, c 3.3 .2$ и $c 3.3 .3$ можно не рассматривать. В случаях $c 3.3 .1 .2$ и $c 3.3 .1 .3$ рассуждения переносятся к пп. 4 и 5 соответственно. После 23.3 .1 .1 возврашаемся к началу п. 7, т.е. в рассуждениях возможна особая последовательность $z_{3}=\{c 3.3 .1 .1, c 3.2 .2, c 2.2\}$. После последовательности $\left[z_{3}\right]_{n}$, $n \in \mathbb{N}$, может идти вариант $c 3.1 .1$.

7.2. Пусть $\bar{c}=\{c 3.3 .1 .1, c 3.2 .1\}$. Параметр $\operatorname{par}(\bar{c})=\operatorname{par}_{c 3.2 .1}(c 3.3 .1 .1)+$ $[3,2]=[6,3]$, и далее переходим к $c 1.1$ или $c 1.2$ с общим параметром последовательности $[7,4]$. 
7.3. Пусть, наконец, $\bar{c}=\{c 3.3 .1 .1, c 3.1 .1\}$. Параметр $\operatorname{par}(\bar{c})=[4,1]+[3,1]=$ $[7,2]$, и ниже постараемся рассуждать как можно точнее.

7.3.1. Допустим, продолжением $\bar{c}$ является случай $c 2.1$. Имеем $g(\{\bar{c}, c 2.1\})=$ $\left(a x_{5} \ldots, d a b y_{2} \ldots\right)$. Отсюда следует, что дальнейший возможный случай - это $c 1.1$ ( $c 1.2$ быть не может) и

$$
\operatorname{par}(\{\bar{c}, c 2.1, c 1.1\})=[7,2]+[1,1]+[1,1]=[9,4] .
$$

Продолжим этот случай.

7.3.1.1. Пусть последовательность случаев $c^{\prime}=\{\bar{c}, c 2.1, c 1.1\}$ продолжается случаем $c 2.1$. Тогда параметр $\operatorname{par}\left(\left\{c^{\prime}, c 2.1\right\}\right)=[10,5]$, и далее мы переходим или к $c 1.1$, или $c 1.2$, что дает параметр последовательности вариантов $\mathrm{par}=[11,6]$, и мы добились понижения коэффициента $\lambda$.

7.3.1.2. Рассмотрим последовательность $\widetilde{c}=\left\{c^{\prime}, c 2.2\right\}$. Нетрудно проверить, что $g(\widetilde{c})=\left(a x_{6} \ldots, a b a y_{2} \ldots\right)$, и, таким образом, среди подслучаев $c 3$ мы вынужденно переходим к случаю $c 3.1 .2$. Так как $\operatorname{par}_{c 3.1 .2}(c 2.2)=[0,1]$, то

$$
\operatorname{par}(\{\widetilde{c}, c 3.1 .2\})=[9,4]+[0,1]+[3,1]=[12,6]
$$

и для последующих продолжений (принимая во внимание, что $\lambda=12 / 6=2$ ) мы можем сослаться на п. 3 с началом $c 3.1 .2$.

7.3.2. Пусть продолжение $\bar{c}$ - это случай $c 2.2$. Аналогично п. 7.3.1 имеем

$$
g(\{\bar{c}, c 2.2\})=\left(a x_{5} \ldots, \operatorname{adabay}_{2} \ldots\right),
$$

поэтому следующий переход возможен лишь к случаям $c 3.2 .1, c 3.1 .1$ или $c 3.2 .2$.

Рассмотрим каждый из этих переходов.

7.3.2.1. $c^{\prime}=\{\bar{c}, c 2.2, c 3.2 .1\}$. Используя замечание о снижении длины преобразования, имеем

$$
\operatorname{par}\left(c^{\prime}\right)=\operatorname{par}(\bar{c})+\operatorname{par}_{c 3.2 .1}(c 2.2)+\operatorname{par}(c 3.2 .1)=[7,2]+[3,3]=[10,5] .
$$

После неизбежного перехода далее к случаям $c 1.1$ или $c 1.2$ мы получаем параметр последовательности $[11,6]$, и тем самым понижение коэффициента $\lambda=11 / 6<2$ произошло.

7.3.2.2. $c^{\prime}=\{\bar{c}, c 2.2, c 3.1 .1\}$. Мы имеем несколько возможных путей построения последовательности. Первый из них - цикл на основе последовательности $z_{2}$. Как будет показано ниже, большое число повторений данного цикла влечет понижение коэффициента $\lambda$ последовательности до числа $<2$, поэтому (предполагаем худшее) должен произойти выход из цикла.

В связи с этим особенно интересны другие возможности.

7.3.2.2.1. Разрыв цикла после $c 3.1 .1$ или просто последовательность $c^{\prime}$ продолжена случаем $c 2.1$. Параметр последовательности $\overline{\bar{c}}=\{c 3.3 .1 .1, c 3.1 .1, c 2.2$, $c 3.1 .1, c 2.2, \ldots, c 3.1 .1, c 2.1\}$ равен $[4+2 n, 1+n]$, где $n-$ количество случаев $c 3.1 .1$, а текущий элемент $g(\overline{\bar{c}})=\left(a x_{2 n+2} \ldots\right.$, dabay $\left._{2} \ldots\right)$.

Нетрудно видеть, что наша последовательность равносильна (в данном контексте) последовательности $\{c 3.3 .1 .1, c 3.1 .1, c 2.1\}$, а значит, можно сослаться на п. 7.3.1.

7.3.2.2.2. Пусть разрыв цикла произошел после случая $c 2.2$. Аналогично предыдушему пункту, учитывая параметр и вид элемента $\bar{g}$ для нашей последовательности, можно считать, что перед нами последовательность $\{c 3.3 .1 .1, c 3.1 .1, c 2.2\}$ без 
перехода к c3.1.1. Таким образом, нам надо продолжать рассматривать п. 7.3.2, минуя п. 7.3.2.2.

7.3.2.3. Пусть $c^{\prime}=\{\bar{c}, c 2.2, c 3.2 .2\}$. Отметим, что

$$
\operatorname{par}\left(c^{\prime}\right)=\operatorname{par}(\bar{c})+\operatorname{par}(\{c 2.2, c 3.2 .2\})=[7,2]+[4,3]=[11,5]
$$

и $g(c 3.2 .2)=\left(x_{2} a \ldots, a d y_{2} a y_{3} \ldots\right)$, поэтому $\bar{g}\left(c^{\prime}\right)=\left(x_{6} a \ldots, a c a y_{3} \ldots\right)$.

7.3.2.3.1. Если далее последовательность продолжается случаем $c 2.1$, то мы имеем последовательности $\left\{c^{\prime}, c 2.1, c 1.1\right\}$ или $\left\{c^{\prime}, c 2.1, c 1.2\right\}$. Параметр любой из этих последовательностей равен $[11,5]+[1,1]+[1,1]=[13,7]$. Отсюда получаем коэффициент $\lambda<2$.

7.3.2.3.2. Осталось рассмотреть последовательность вида $\overline{\bar{c}}=\left\{c^{\prime}, c 2.2\right\}$. Имеем $\bar{g}(\overline{\bar{c}})=\left(a x_{7} \ldots, a_{a c a y_{2}} \ldots\right)$, поэтому после $c 2.2$ возможны переходы к вариантам $c 3.1 .1, c 3.2 .1$ или $c 3.3$. Учитывая возможные сокрашения:

$$
\operatorname{par}_{c 3.2 .1}(c 2.2)=\operatorname{par}_{c 3.3 .2}(c 2.2)=\operatorname{par}_{c 3.3 .3}(c 2.2)=[0,1]
$$

можно считать, что в этих случаях $\operatorname{par}(\overline{\bar{c}})=\operatorname{par}\left(c^{\prime}\right)+[0,1]=[11,6]$.

При переходе к варианту с3.3.1.1 может возникнуть особая последовательность

$$
z_{4}=\{c 3.3 .1 .1, c 3.1 .1, c 2.2, c 3.2 .2, c 2.2\}
$$

При переходе же к случаям $c 3.1 .1, c 3.3 .1 .2$ или $c 3.3 .1 .3$ можно считать, что $\operatorname{par}(\overline{\bar{c}})=$ $[12,6]$, и сослаться на последовательности с соответствующими началами, рассмотренньми в пाп. 6,4 и 5 .

Мы показали, что коэффициент $\lambda$ любой достаточно длинной возможной последовательности случаев становится меньше 2 всегда, кроме, быть может, вариантов зацикливания в особых последовательностях.

Согласно рассуждениям, приведенньм выше, перечислим возможные особые последовательности:

а) $z_{1}=\{c 3.1 .2, c 1.2\}$

б) $z_{2}=\{c 3.1 .1, c 2.2\}$,

в) $z_{3}=\{c 3.3 .1 .1, c 3.2 .2, c 2.2\}$,

г) $z_{4}=\{c 3.3 .1 .1, c 3.1 .1, c 2.2, c 3.2 .2, c 2.2\}$.

Предположим, мы имеем пару $\left(g_{1}, g_{2}\right)$, которая индуцирует цикл $\left[z_{1}\right]_{4}$. Тогда $g_{2}=(a b)^{2^{3}} a y_{9} a \ldots$ Но $|a b|=16$ и, значит, перед нами неприведенное слово, вопреки предположению. Таким образом, последовательность $z_{1}$ не может повторяться подряд более трех раз и должен произойти выход из цикла. При выходе из цикла мы можем сослаться на дальнейшие рассуждения о последовательностях случаев, минуя последовательность $z_{1}$.

По аналогичной причине для приведенного слова $g_{1}$ циклы на основе последовательностей $z_{2}, z_{3}$ или $z_{4}$ должны также прерываться. Однако, как мы видели выше, последовательности $z_{2}, z_{3}$ и $z_{4}$ могут следовать одна за другой. Рассмотрим эту возможность.

Пусть начала слов $g_{1}$ и $g_{2}$ таковы, что для нашего процесса необходим один из вариантов $z_{2}, z_{3}$ или $z_{4}$. Рассмотрим $\psi\left(g_{1}\right)=\left(g_{2,1}, g_{2,2}\right)$. Легко видеть, что для варианта $z_{2}$ начало слова $g_{2,2}$ - это $a c$, мы имеем $g_{2,2}=(a c)^{2} \ldots$ для $z_{3}$ и $g_{2,2}=(a c)^{3} \ldots$ для $z_{4}$. В соответствии со степенью элемента $a c$ положим

$$
p\left(z_{2}\right)=1, \quad p\left(z_{3}\right)=2, \quad p\left(z_{4}\right)=3 .
$$


Предположим, что слова $g_{1}$ и $g_{2}$ начиная с некоторого момента индуцируют последовательность случаев вида $z_{i_{1}}, z_{i_{2}}, \ldots$, где $i_{j} \in\{2,3,4\}$. Так как $|a c|=8$, то в этом случае слово $g_{2,2}$ ограничено по длине.

Так как фрагменты слов $g_{1}$ или $g_{2}$ однозначно задают последовательность вариантов наших рассуждений в лемме 1 и как было видно выше, для задания особой последовательности достаточно фрагмента слова $g_{1}$ (или $g_{2}$ ).

$\Phi$ рагмент слова $g_{1}$, индуцируюший последовательность случаев $z_{i_{1}}, z_{i_{2}}, \ldots, z_{i_{s}}$

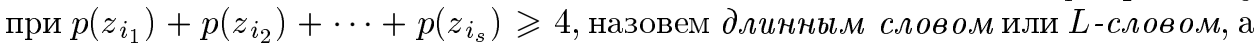
при сумме $<4-$ коротким словом или sh-словом. При этом будем считать, что слово $g_{2}$ - любое возможное для данных вариантов.

Так как $g \notin F_{1 / 6}^{* *}(n)$, то $g_{2,2}$ не может быть ограничено по длине с ростом длины $g$. Поэтому $L$-слово в слове $g_{1}$ должно обрываться. Разобьем слово $g_{1}$ на фрагменты, каждый из которых является $L$-словом, sh-словом или обычным словом, не содержащим особую последовательность. Пусть $m_{1}, m_{2}$ и $m_{3}$ - общие длины соответственно всех $L$-слов, sh-слов и обычных слов в слове $g_{1}$.

Так как максимальное по длине sh-слово ограничено длиной 15 и $L$-слово, записанное рядом c sh-словом, дает одно $L$-слово, то в слове $g_{1}$ возможно следующее чередование фрагментов: $\ldots x z y z \ldots, \ldots y z x z \ldots$, где $x-L$-слово, $y-$ sh-слово и $z$ - обыгное слово.

Пусть $\lambda^{\prime}$ - наибольший коэффициент последовательности случаев, индуцируемой обычньм словом. Как было показано выше, $\lambda^{\prime}<2$. Пусть $k$ - число $L$-слов в слове $g_{1}$. Отсюда наибольший общий параметр слова $g$ можно оценить как

$$
\operatorname{par}(g)=\left[u_{1}, u_{2}\right] \leqslant[30 k, 15 k]+\left[\lambda^{\prime} m_{2}, m_{2}\right]+\left[2 m_{1}, m_{1}\right]+\left[\lambda^{\prime} m_{2}, m_{2}\right] .
$$

С другой стороны, каждое $L$-слово увеличивает длину слова $g_{2,2}$ не более, чем на 3.

Обозначим $m=m_{2}+m_{3}$. Так как при $\mu \leqslant 1 / 6$ имеем $g \notin F_{\mu}^{* *}(n)$, то

$$
l\left(g_{2,2}\right) \geqslant \frac{1}{2(1+\mu)} l\left(g_{1}\right)
$$

Однако $l\left(g_{2,2}\right) \leqslant 3 k+m / 2$ и мы имеем

$$
\frac{m+m_{1}}{2(1+\mu)} \leqslant 3 k+\frac{m}{2}
$$

Для $\mu=1 / 6$ получаем

$$
m_{1} \leqslant 7 k+\frac{m}{6}
$$

Наименьшая длина $L$-слова равна 8 и, значит, $m_{1} \geqslant 8 k$. Отсюда $k \leqslant m / 6$ и $m_{1} \leqslant 4 m / 3$.

Обозначим $\lambda(g)=u_{1} / u_{2}$. Из рассуждений, приведенных выше, делаем вывод, что

$$
\lambda(g) \leqslant \frac{30 k+2 m_{1}+2 \lambda^{\prime} m_{2}}{15 k+m_{1}+2 m_{2}} .
$$

Эта дробь будет наибольшей при максимально больших $k$ и $m_{1}$ и малых $m_{2}$. Используя неравенство $m_{2} \geqslant m-15 k$ и неравенства, полученные выше, имеем

$$
\lambda(g) \leqslant \frac{30 k+\frac{8}{3} m+2 \lambda^{\prime}(m-15 k)}{15 k+\frac{4}{3} m+2(m-15 k)}=\frac{\frac{8}{3} m+2 \lambda^{\prime} m-\left(\lambda^{\prime}-1\right) \cdot 30 k}{\frac{4}{3} m+2 m-15 k} .
$$


Так как $\lambda^{\prime}>1$ и $m$ можно выбрать сколь угодно большим, то последняя дробь допускает оценку

$$
\lambda(g) \leqslant \frac{\frac{8}{3}+2 \lambda^{\prime}}{\frac{4}{3}+2}=\frac{4+3 \lambda^{\prime}}{5}<2 .
$$

С поправкой на короткие слова $g_{1}$ и $g_{2}$ выберем константу $L_{1}$, не превосходящую 16 .

Для произвольного слова $g$ из множества, определенного в лемме, мы показали, что

$$
l(g) \leqslant \lambda(g)\left(l\left(g_{1}\right)+l\left(g_{2}\right)\right)+L_{1}
$$

и лемма 2 доказана.

Для получения оценки (2) отметим, что можно положить

$$
Q=\max \left\{\frac{4+3 \lambda^{\prime}}{5} \cdot 2, \sqrt{\frac{38}{2}}\right\}<4 .
$$

Лемма 1 доказана.

\section{§ 3. Нахождение параметра $\lambda^{\prime}$}

Мы показали, что верна лемма 1 с параметром $Q<4$. При этом выбор $Q$ зависит от некоторого $\lambda^{\prime}$. Для полного доказательства теоремы укажем теперь конкретные $\lambda^{\prime}$ и $Q$.

Пользуясь рассуждениями леммы 2 , параметр любого слова, не являющегося $L$-словом или sh-словом, в зависимости от варианта представлен в таблице 4.

ТАБЛИЦА 4

\begin{tabular}{|l|l|}
\hline Вариант & Максимальный параметр \\
\hline 1 & {$[9,5]$} \\
\hline 2 & {$[7,4]$} \\
\hline 3 & {$[4,2]+$ п. $1=[13,7]$} \\
\hline 4 & {$[7,4]$} \\
\hline 5 & {$[7,4]$} \\
\hline 6 & {$[4,2]+$ п. 4 или п. $5=[11,6]$} \\
\hline 7.1 & п. 4 или п. $5=[7,4]$ \\
\hline 7.2 & {$[7,4]$} \\
\hline 7.3 .1 .1 & {$[11,6]$} \\
\hline 7.3 .1 .2 & {$[10,5]+$ п. $3=[23,12]$} \\
\hline 7.3 .2 .1 & {$[11,6]$} \\
\hline 7.3 .2 .2 .1 & п. $7.3 .1 .2=[23,12]$ \\
\hline 7.3 .2 .2 .2 & {$[11,6]$} \\
\hline 7.3 .2 .3 .1 & {$[13,7]$} \\
\hline 7.3 .2 .3 .2 & п. 4 или п. $5=[7,4]$ \\
\hline
\end{tabular}

Итак, наибольший совокупный коэффициент $\lambda^{\prime}$ может быть выбран $\leqslant 23 / 12$. Отсюда

$$
\lambda \leqslant \frac{4+3 \cdot \frac{23}{12}}{5}=\frac{39}{20}, \quad Q=3.9
$$


Имеем

$$
\gamma_{G}(n) \succeq e^{n^{\alpha}}
$$

где

$$
\alpha \geqslant \log _{Q} 2=\log _{3.9} 2>\frac{1}{2}
$$

Теорема полностью доказана.

Отметим, что Л. Бартольди [13], используя основную идею автора, предложил недавно иное доказательство нашей теоремы.

Автор выражает глубокую благодарность Р.И. Григорчуку за постановку задачи и ценные замечания.

\section{Список литературы}

1. Григорчук Р. И. К проблеме Бернсайда о периодических группах // Функц. анализ и его прилож. 1980. Т. 14. №1. С. 53-54.

2. Григорчук Р. И. Пример конечно определенной аменабельной группы, не принадлежащей классу $E G$ // Матем. сб. 1998. Т. 189. №1. С. 79-100.

3. de la Harpe P. Topics in geometric group theory // Preprint, http://www.unige.ch/math/ biblio/preprint/pp98.html.

4. Milnor J. Problem 5603 // Amer. Math. Monthly. 1968. V. 75. №6. P. 685-686.

5. Григорчук Р.И. Степени роста конечно порожденных групп и теория инвариантных средних // Изв. АН СССР. Сер. матем. 1984. Т. 48. № 5. С. 939-985.

6. Григорчук Р. И. О ряде Гильберта-Пуанкаре градуированных алгебр, ассоциированных с группами // Матем. сб. 1989. Т. 180. № 2. С. 207-225.

7. Klaas G., Leedham-Green C. R., Plesken W. Linear pro-p-groups of finite width. Berlin: Springer-Verlag, 1997. (Lecture Notes in Math. V. 1674.)

8. Bartholdi L., Grigorchuk R. I. Lie methods in growth of groups and groups of finite width // Computational and geometric aspects of modern algebra. Proceedings of the workshop, Edinburgh, UK, July 23-31, 1998 / ed. M. Atkinson et al. Cambridge: Cambridge Univ. Press, 2000. P. 1-27.

9. Grigorchuk R. I. On growth in group theory // Proceedings of the International Congress of Mathematicians. Kyoto, 1990. V. I. Tokyo: Math. Soc. Japan, 1991. P. 325-338.

10. Leonov Yu. G. On growth function for some torsion residually finite groups // Abstr. Pontrjagin Int. Math. Conf. Moscow, 1998. P. 36-38.

11. Рожков А. В. Условия конечности в группах автоморфизмов деревьев // Дис. ... докт. физ.-матем. наук. Челябинский гос. ун-т, 1996.

12. Леонов Ю. Г. Проблема сопряженности в одном классе 2-групп // Матем. заметки. 1998. Т. 64. № 4. C. 573-583.

13. Bartholdi L. Lower bounds on the growth of Grigorchuk's torsion group // Preprint, 2000.

Украинская государственная академия связи, г. Одесса, Украина

Поступила в редакцию

E-mail: leo@farlep.net, leonov@visto.com

26.12 .2000 